\title{
Acromesomelic Dysplasia with Interstitial Lung Disease: A Unique Association
}

\author{
${ }^{1}$ Chinnu Sasikumar, ${ }^{2}$ Ketaki Utpat, ${ }^{3}$ Unnati Desai, ${ }^{4}$ Jyotsna M Joshi
}

\begin{abstract}
Acromesomelic dysplasia is an extremely uncommon skeletal dysplasia with an autosomal recessive inheritance. It is characterized by a constellation of skeletal anomalies. Respiratory impediments have been sporadically reported earlier in various skeletal dysplasias. However, respiratory affection in acromesomelic dysplasia has not been elucidated earlier. We herein report a case of acromesomelic dysplasia associated with interstitial lung disease (ILD). Diagnosis of acromesomelic dysplasia was based on radiographs of whole skeleton.
\end{abstract}

Keywords: Acromesomelic dysplasia, Interstitial lung disease, Skeletal dysplasia.

How to cite this article: Sasikumar C, Utpat K, Desai U, Joshi JM. Acromesomelic Dysplasia with Interstitial Lung Disease: A Unique Association. Int J Recent Surg Med Sci 2018;4(1):29-31.

\section{Source of support: Nil}

Conflict of interest: None

\section{INTRODUCTION}

Skeletal dysplasias are disorders of cartilage and bone leading to a generalized abnormality in the skeleton. They constitute a heterogeneous group differing in their natural histories, inheritance patterns, etiopathogenesis, and prognosis. ${ }^{1}$ Pulmonary disorders, such as dysgammaglobulinemia, severe recurrent pneumonias, bronchiectasis, and asthma, have been seen to coexist with skeletal dysplasias. Acromesomelic dysplasia describes a group of extremely rare, inherited, autosomal recessive progressive skeletal conditions that result in a particular form of short stature, called short limb dwarfism. The short stature is the result of unusually short forearms and forelegs (mesomelia) and abnormal shortening of the bones in the hands and feet (acromelia). ${ }^{1}$ It is characterized by severe shortening of middle and distal segments of limbs but normal intelligence and facial

\footnotetext{
${ }^{1}$ Junior Resident, ${ }^{2,3}$ Assistant Professor, ${ }^{4}$ Head

${ }^{1-4}$ Department of Pulmonary Medicine, Topiwala National Medical College and BYL Nair Charitable Hospital, Mumbai Maharashtra, India

Corresponding Author: Jyotsna M Joshi, Head, Department of Pulmonary Medicine, Topiwala National Medical College \& BYL Nair Charitable Hospital, Mumbai, Maharashtra, India, Phone: +912223003095, e-mail: drjoshijm@gmail.com
}

appearance. Various lung manifestations have been sporadically reported to be associated with the condition. These include bronchiectasis, tracheomalacia, tracheal stenosis, and obstructive sleep apnea. ${ }^{2}$ However, ILD in association with acromesomelic dysplasia has not been reported earlier.

\section{CASE REPORT}

A 50-year-old man, occasional smoker presented with complaints of dry cough and exertional breathlessness since 1 year. There was no history of exposure to organic or inorganic dust, joint pain, photosensitivity or Raynaud's phenomenon. There was a history of corneal opacity since childhood. On clinical examination, there was grade I clubbing. He was $140 \mathrm{~cm}$ tall with body mass index of $18 \mathrm{~kg} / \mathrm{m}^{2}$. The patient had a broad forehead, small flat nose, normal head circumference, shortened bilateral forearms (Fig. 1), ulnar deviation of hands with limited elbow extension (Fig. 1), left bifid thumb (Fig. 2), corneal opacity in the right eye (Fig. 3), and shortened left foreleg (Fig. 4). Spinal deformity in the form of scoliosis with convexity to right side with decreased spinoscapular distance on right side was appreciated (Fig. 4). He had bilateral end inspiratory fine basilar crackles on auscultation. Cardiovascular, abdominal, and neurologic examinations were unremarkable. Patient had normal intelligence. Chest radiograph showed bilateral reticulonodular opacities. Arterial blood gas (ABG) estimation showed pH 7.38, $\mathrm{pCO}_{2} 42 \mathrm{~mm} \mathrm{Hg}, \mathrm{pO}_{2} 70 \mathrm{~mm}$ $\mathrm{Hg}, \mathrm{HCO}_{3} 34.2 \mathrm{mmol} / \mathrm{L}$, and arterial oxygen saturation

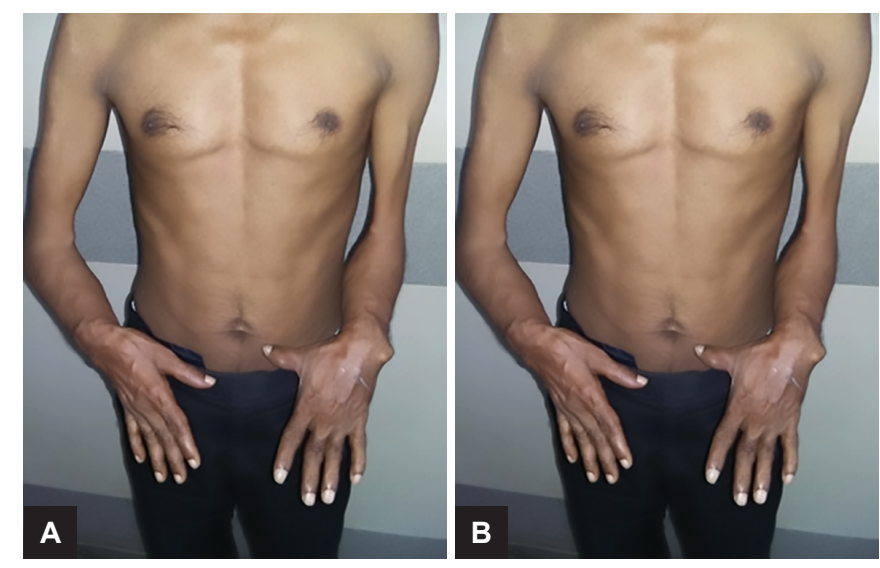

Figs $1 \mathrm{~A}$ and B: Shortened bilateral forearms with ulnar deviation of hands 


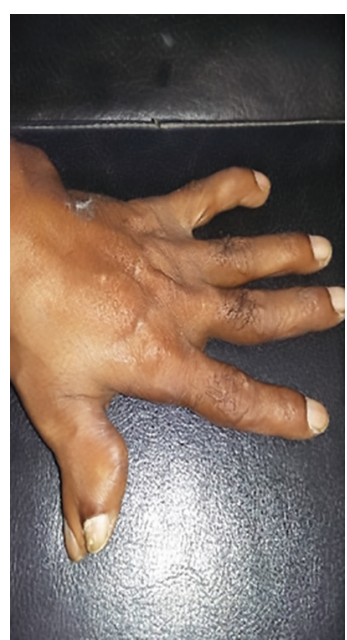

Fig. 2: Left bifid thumb

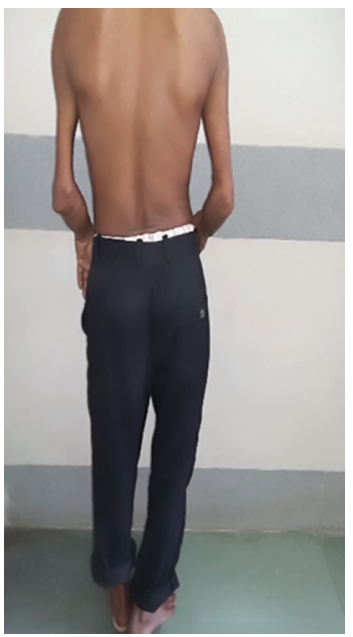

Fig. 4: Kyphoscoliosis with shortened left leg

$96 \%$. On investigation, hemoglobin was $12 \mathrm{gm} / \mathrm{dL}$, total leukocyte count was $8,500 \mathrm{~mm}$, with neutrophils $62 \%$ and lymphocytes $38 \%$. Other biochemical parameters were within normal limits. Spirometry was within normal

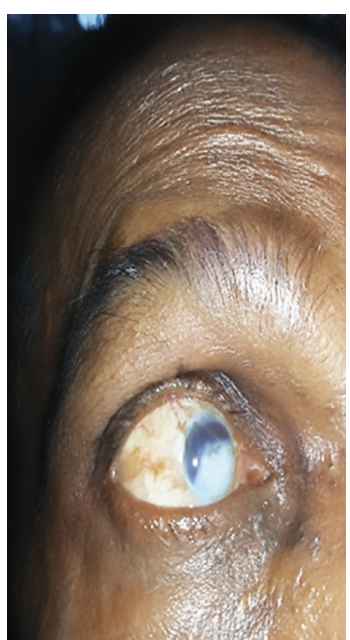

Fig. 3: Right corneal opacity

limits. Patient's 6-minute walk distance was $340 \mathrm{~m}$ and he did not desaturate postexercise. High-resolution computed tomography (CT) thorax revealed interstitial septal thickening, honey combing and traction bronchiectasis predominantly in bilateral lower lobe areas consistent with ILD, usual interstitial pneumonia (UIP) pattern (Fig. 5). Two-dimensional echocardiography showed mild pulmonary hypertension. Skeletal survey performed through X-ray long bones of both upper and lower limbs revealed mesomelic shortening of the upper extremity with lesser involvement of lower extremity. Radius was short and bowed with long and curved ulna on both sides. Presence of a supernumerary distal phalanx of first digit on left and short left femoral head was also reported. The final diagnosis was ILD with idiopathic pulmonary fibrosis associated with acromesomelic dysplasia. The patient was kept under observation as lung functions were within normal limits. Patient was followed up and assessed every 3 months with spirometry, ABG, 6-minute walk distance.
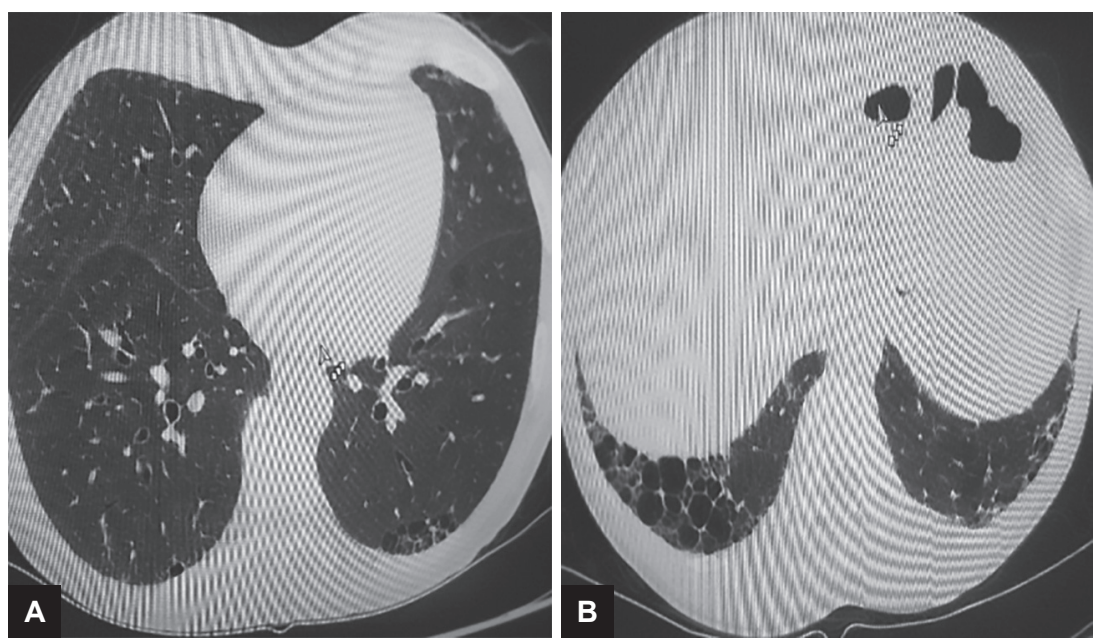

Figs 5A and B: The CT thorax showing interstitial septal thickening, traction bronchiectasis, honeycombing in bilateral lower lobe areas 


\section{DISCUSSION}

Acromesomelic dysplasia is a rare, inherited, progressive skeletal disorder that results in a particular form of short stature known as short limb dwarfism. This disorder is characterized by acromelia and mesomelia. Mesomelia is referred to as the shortening of the bones of the forearms and lower legs relative to the upper parts of upper and lower limbs, whereas acromelia is the shortening of the bones of the hands and feet. Therefore, the short stature of affected individuals is the result of unusually short forearms and abnormal shortening of bones of the lower legs. Acromesomelic dysplasia is of three types: (i) Maroteaux type (AMDM); (ii) Hunter-Thompson type; and (iii) Grebe type. ${ }^{3}$ Maroteaux type is an autosomal recessive skeletal disorder that usually affects the limbs and the spine. Newborns affected with AMDM can have short appearing limbs but weight, length, and head circumference would be normal. This seems to occur because of premature fusion of metaphyses of certain long bones. In Hunter-Thompson type, adult height, facial appearance, head circumference, and intelligence are normal and trunk is normally proportioned. However, the upper and lower limbs are markedly shortened, with distal segments more affected than the proximal segments. ${ }^{4}$ Such patients may have frequent dislocations and restricted movements of large joints. In the majority of cases, fingers are short and of unequal length, but are symmetric between both hands. Deformities are more common in lower limbs than the upper limbs. In Grebe type of acromesomelic dysplasia, adult height is below $100 \mathrm{~cm}$, with normal axial skeleton, severe hypomelia and proximal segments more affected than distal. ${ }^{5}$ Our patient probably had Hunter-Thompson type of dysplasia as axial skeleton was spared and distal segment was more affected than the proximal. In most cases, in addition to short, broad hands and feet, affected infants often have normal weight and characteristic facial abnormalities, such as enlarged head, prominent forehead, pronounced back portion of the head, a slightly flattened midface, and/or an abnormally small, pug nose. During the first years of life, short stature begins to become apparent as the forearms, lower legs, hands, and feet do not grow proportionally with the rest of the body. Due to abnormal development and premature fusion (ossification) of the shafts of the long bones of the arm, bones of forearm (ulna and radius) may be markedly shortened and abnormally curved. In addition, the end portion of the radius that meets with the humerus to form part of the elbow joint may be completely or partially dislocated (subluxation), and this is known as Madelung deformity. As a result, supination, pronation, and full extension of arms become difficult. Some affected individuals may also experience stiffness, tenderness, and pain of the elbows suggestive of osteoarthritis. During early childhood, individuals with AMD may also begin to demonstrate vertebral abnormalities, such as low thoracic kyphosis and lower spine lumbar hyperlordosis.

Reported lung manifestations of skeletal dysplasias include bronchiectasis, tracheomalacia, tracheal stenosis, and obstructive sleep apnea. ${ }^{2,5}$ However, to the best of our knowledge, ILD associated with skeletal dysplasia has not been reported earlier in literature. Our case had a skeletal dysplasia in the form of acromesomelic dysplasia and an associated ILD with UIP pattern. There has been only a single case report documenting the respiratory manifestation of acromesomelic dysplasia in the form of bronchiectasis. ${ }^{5}$ And this is the second case bringing to light another respiratory association of the disorder in the form of an ILD. Whether this association is causal due to some unexplored genetic linkage or incidental is a matter of speculation. Genetic analysis is warranted to unravel a common linkage which could be contributory toward skeletal anomalies and lung involvement.

\section{REFERENCES}

1. Teja SR, Manne B, Mahita BD. Skeletal dysplasias-clinicoradiological review. Int J Sci Stud 2016 Nov;4(8):223-229.

2. Catena E, Bariffi F, Virenzio L. On an unusual case of diffuse chondrodysplasia of the bronchial walls. Arch Tisiol Mal Appar Respir 1968 Oct;23(10):759-782.

3. Superti-Furga A, Bonafe L, Rimion DL. Molecular pathogenetic classification of genetic disorders of skeleton. Am J Med Genet 2002 Winter;106(4):282-293.

4. Langer LO, Cervenka J, Camargo M. A severe autosomal recessive acromesomelic dysplasia, the Hunter-Thompson type, and comparison with the Grebe type. Hum Genet 1989 Mar;81(4):323-328.

5. Farnaz S, Gothi D, Joshi JM. Acromesomelic dysplasia with bronchiectasis. Indian J Chest Dis Allied Sci 2005 Apr-Jun;47:131-134. 